\title{
Subsolid Pulmonary Nodule
}

National Cancer Institute

\section{Source}

National Cancer Institute. Subsolid Pulmonary Nodule. NCI Thesaurus. Code C138144.

A term that refers to a pure ground-glass pulmonary nodule or a part-solid pulmonary nodule on CT scan examination. 